\title{
Multiplicity results for sublinear elliptic equations with sign-changing potential and general nonlinearity
}

Wei $\mathrm{He}^{1}$ and Qingfang Wu 2* $^{*}$

"Correspondence:

212032@csu.edu.cn

${ }^{2}$ School of Traffic and

Transportation Engineering, Central South University, Changsha, 410075

Hunan, P.R. China

Full list of author information is

available at the end of the article

\section{Springer}

\section{Abstract}

In this paper, we study the following elliptic boundary value problem:

$$
\left\{\begin{array}{l}
-\Delta u+V(x) u=f(x, u), \quad x \in \Omega, \\
u=0, \quad x \in \partial \Omega,
\end{array}\right.
$$

where $\Omega \subset \mathbb{R}^{N}$ is a bounded domain with smooth boundary $\partial \Omega$, and $f$ is allowed to be sign-changing and is of sublinear growth near infinity in $u$. For both cases that $V \in L^{N / 2}(\Omega)$ with $N \geq 3$ and that $V \in C(\Omega, \mathbb{R})$ with $\inf _{\Omega} V(x)>-\infty$, we establish a sequence of nontrivial solutions converging to zero for above equation via a new critical point theorem.

MSC: $35 J 25 ; 35 J 60$

Keywords: Semilinear elliptic equations; Boundary value problems; Sublinear; Indefinite sign; Genus

\section{Introduction}

Consider the elliptic boundary value problem

$$
\left\{\begin{array}{l}
-\Delta u+V(x) u=f(x, u), \quad x \in \Omega \\
u=0, \quad x \in \partial \Omega
\end{array}\right.
$$

where $V: \Omega \rightarrow \mathbb{R}$ and $f: \Omega \times \mathbb{R} \rightarrow \mathbb{R}, \Omega \subset \mathbb{R}^{N}$ is a bounded domain with smooth boundary $\partial \Omega$.

The semilinear elliptic equation has found a great deal of interest in the past several decades. With the aid of variational methods, existence and multiplicity of nontrivial solutions for problem (1.1) have been extensively studied under various assumptions on the potential $V(x)$ and the nonlinearity $f(x, u)$; see $[1,2,5,10,11,13,15-18,20,25,26,28-32]$ and the references therein.

(c) The Author(s) 2020. This article is licensed under a Creative Commons Attribution 4.0 International License, which permits use, sharing, adaptation, distribution and reproduction in any medium or format, as long as you give appropriate credit to the original author(s) and the source, provide a link to the Creative Commons licence, and indicate if changes were made. The images or other third party material in this article are included in the article's Creative Commons licence, unless indicated otherwise in a credit line to the material. If material is not included in the article's Creative Commons licence and your intended use is not permitted by statutory regulation or exceeds the permitted use, you will need to obtain permission directly from the copyright holder. To view a copy of this licence, visit http://creativecommons.org/licenses/by/4.0/. 
It is well known that weak solutions to (1.1) correspond to critical points of the energy functional:

$$
\Phi(u)=\frac{1}{2} \int_{\Omega}\left(|\nabla u|^{2}+V(x) u^{2}\right) \mathrm{d} x-\int_{\Omega} F(x, u) \mathrm{d} x,
$$

where here and in the sequel $F(x, t):=\int_{0}^{t} f(x, s) \mathrm{d} x$. For the case of $\inf _{\Omega} V>-\lambda_{1}(\Omega), 0$ is a local minimum of $\Phi$, techniques based on the mountain-pass theorem have been well applied; see $[1,17,26,30]$. When $\inf _{\Omega} V \in\left(-\infty,-\lambda_{1}(\Omega)\right), 0$ is a saddle point rather than a local minimum of $\Phi$. Problem (1.1) is indefinite, and the main obstacle is to establish the boundedness of the Palais-Smale sequence for $\Phi$. Under assumptions that $V \in L^{N / 2}(\Omega)$ with $N \geq 3$ and $f$ is superlinear near infinity in $u, \mathrm{Li}$ and Willem [15] obtained one nontrivial solution via a local linking method; see also Willem [30] via the linking theorem [26]. Later, this result was improved by Jiang and Tang [11] under a weak superquadratic condition introduced by Costa [5, 6]; see also [18], where local linking and symmetric mountain-pass theorem were also used. In [31], infinitely many solutions of (1.1) was proved by Zhang and Liu by using the variant fountain theorem established in [33]. With the aid of symmetric mountain-pass lemma, this result was improved and generalized by Qin et al. in [25]; see also [13] for similar results. Recently, under some new superquadratic conditions on $f$, Tang [29] showed that (1.1) has a ground state solution, as well as infinitely many pairs of solutions provided that $V$ satisfies

(V) $V \in C(\Omega, \mathbb{R})$ and $\inf _{\Omega} V(x)>-\infty$.

The existence of infinitely many nontrivial solutions was obtained by He and Zou [10] for the case that $f$ asymptotically shows linear growth near infinity. For related topics including the case of an unbounded domain, we refer the reader to $[3,4,6,7,9,12,14,16,19,21-$ $24,27,28]$ and the references therein.

In [32], Zhang and Tang, via the variant fountain theorem established in [33], first studied the sublinear case provided that $V \in L^{N / 2}(\Omega)$ and $f$ satisfies:

(F1) $F \in C^{1}(\Omega \times \mathbb{R},[0, \infty))$, and there exist constants $\mu \in[1,2)$ and $r_{1}>0$ such that

$$
f(x, u) u \leq \mu F(x, u), \quad \forall x \in \Omega,|u| \geq r_{1}
$$

(F2) $\lim _{|u| \rightarrow 0} \frac{F(x, u)}{|u|^{2}}=\infty$ uniformly for $x \in \Omega$, and there exist constants $c_{1}, r_{2}>0$ such that

$$
F(x, u) \leq c_{1}|u|, \quad \forall x \in \Omega,|u| \leq r_{2}
$$

(F3) there exists a constant $d>0$ such that

$$
\liminf _{|u| \rightarrow \infty} \frac{F(x, u)}{|u|} \geq d \quad \text { uniformly for } x \in \Omega
$$

Specifically, the following theorem was established [32].

Theorem 1.1 ([32]) Suppose that (F1)-(F3), $V \in L^{N / 2}(\Omega)$ with $N \geq 3$ and that $F(x, u)$ is even in $u$ hold. Then problem (1.1) possesses infinitely many nontrivial solutions.

Inspired by the aforementioned work, in the present paper, we continue to study the sublinear case under the following mild assumptions: 
(S1) $F \in C^{1}(\Omega \times \mathbb{R}, \mathbb{R})$, and there exist constants $c>0$ and $p \in(1,2)$ such that

$$
|f(x, u)| \leq c\left(1+|u|^{p-1}\right), \quad \forall(x, u) \in \Omega \times \mathbb{R}
$$

(S2) $F(x, 0)=0$ for all $x \in \Omega$, and

$$
\liminf _{|u| \rightarrow 0} \frac{F(x, u)}{|u|^{2}}=\infty, \quad \text { a.e. } x \in \Omega
$$

(S3) $F(x, u)=F(x,-u), \forall(x, u) \in \Omega \times \mathbb{R}$.

Our main result reads as follows.

Theorem 1.2 Suppose that (S1), (S2), (S3) and that $V \in L^{N / 2}(\Omega)$ or $(\mathrm{V})$ hold. Then problem (1.1) possesses a sequence of nontrivial solutions converging to zero.

Remark 1.3 Nontrivial solutions obtained in Theorem 1.1 is different from ones established in $[4,25,31,32]$, since the sequence of critical points corresponding to the least energy does not necessarily converge to zero; see [12, Example 1.3].

Remark 1.4 Compared with Theorem 1.1, the crucial condition (F2) used in [32] is weakened to (S2), and the technical conditions (F1) and (F3) are got rid of in Theorem 1.2. Moreover, the nonlinearity $f$ considered in this paper is allowed to be sign-changing. Hence, Theorem 1.2 extends and complements related results in [16, 25, 31, 32].

Before proceeding to the proof of main result, we give two examples to illustrate our assumptions.

Example $1.5 F(x, u)=|u|^{3 / 2}\left[\ln 3-\ln \left(1+|u|^{2}\right)\right]$.

It is easy to verify that $F$ satisfies (S1)-(S3), moreover, $F(x, u)<0$ for $|u|>\sqrt{2}$, so (F3) does not hold.

Example 1.6 $F(x, u)=|u|^{1 / 2}(1-|u|)$.

Note that $F(x, u)<0$ for $|u|>1$ and $F$ satisfies (S1)-(S3), but it does not satisfies (F2) and (F3).

\section{Variational setting and proofs of the main results}

As in [29], we introduce the variational framework associated with problem (1.1) under (V) which holds also for the case that $V \in L^{N / 2}(\Omega)$.

Denote by $\mathcal{A}$ the self-adjoint extension of the operator $-\Delta+V$ with domain $\mathfrak{D}(\mathcal{A})$ $\left(C_{0}^{\infty}(\Omega) \subset \mathfrak{D}(\mathcal{A}) \subset L^{2}(\Omega)\right)$. Let $\{\mathcal{E}(\lambda):-\infty \leq \lambda \leq+\infty\}$ and $|\mathcal{A}|$ be the spectral family and the absolute value of $\mathcal{A}$, respectively, and $|\mathcal{A}|^{1 / 2}$ be the square root of $|\mathcal{A}|$. Set $\mathcal{U}=i d-\mathcal{E}(0)-\mathcal{E}(0-)$. Then $\mathcal{U}$ commutes with $\mathcal{A},|\mathcal{A}|$ and $|\mathcal{A}|^{1 / 2}$, and $\mathcal{A}=\mathcal{U}|\mathcal{A}|$ is the polar decomposition of $\mathcal{A}$ (see [8, Theorem IV 3.3]). Let $E=\mathfrak{D}\left(|\mathcal{A}|^{1 / 2}\right)$ and

$$
E^{-}=\mathcal{E}(0-) E, \quad E^{0}=[\mathcal{E}(0)-\mathcal{E}(0-)] E, \quad E^{+}=[\mathcal{E}(+\infty)-\mathcal{E}(0)] E .
$$


For any $u \in E$, it is easy to see that $u=u^{-}+u^{0}+u^{+}$, where

$$
\begin{aligned}
& u^{-}:=\mathcal{E}(0-) u \in E^{-}, \quad u^{0}:=[\mathcal{E}(0)-\mathcal{E}(0-)] u \in E^{0}, \\
& u^{+}:=[\mathcal{E}(+\infty)-\mathcal{E}(0)] u \in E^{+} .
\end{aligned}
$$

Define an inner product

$$
(u, v)=\left(|\mathcal{A}|^{1 / 2} u,|\mathcal{A}|^{1 / 2} v\right)_{L^{2}}+\left(u^{0}, v^{0}\right)_{L^{2}}, \quad \forall u, v \in E,
$$

and the corresponding norm

$$
\|u, v\|=\left\||\mathcal{A}|^{1 / 2} u\right\|_{2}+\left\|u^{0}\right\|_{2}, \quad \forall u \in E
$$

where $(\cdot, \cdot)_{L^{2}}$ denotes the inner product of $L^{2}(\Omega),\|\cdot\|_{s}$ stands for the usual $L^{s}(\Omega)$ norm. Then $E \subset H_{0}^{1}(\Omega)$ is a Hilbert space. Clearly, $C_{0}^{\infty}(\Omega)$ is dense in $E$.

The following lemma was established in [29, Lemmas 2.4, 2.5, Remark 2.8].

Lemma 2.1 Let $(\mathrm{V})$ be satisfied. Then, for the inner products $(\cdot, \cdot)$ and $(\cdot, \cdot)_{L^{2}}$ on $E$, we have

$$
E^{-} \perp E^{0}, \quad E^{-} \perp E^{+}, \quad E^{0} \perp E^{+}, \quad \operatorname{dim}(\mathcal{E}(M) E)<+\infty, \quad \forall M \geq 0,
$$

and

$$
E^{0}=\operatorname{Ker}(\mathcal{A}), \quad \mathcal{A} u^{-}=-|\mathcal{A}| u^{-}, \quad \mathcal{A} u^{+}=|\mathcal{A}| u^{+}, \quad \forall u \in E \cap \mathfrak{D}(\mathcal{A}) .
$$

Moreover, $E$ is compactly embedded in $L^{s}(\Omega)$ for $1 \leq s<2^{*}$, where $2^{*}:=2 N /(N-2)$ if $N \geq 3$ and $2^{*}:=+\infty$ if $N=1$ or 2 .

For the case that $V \in L^{N / 2}(\Omega)$, spectrum of $\mathcal{A}$ consists of only eigenvalues numbered in $-\infty<\mu_{1} \leq \mu_{2} \leq \cdots \leq \mu_{n} \leq 0<\mu_{n+1} \leq \cdots \rightarrow+\infty$ (counted with multiplicity) with the corresponding system of eigenfunctions $\left\{e_{n}\right\}$ forming an orthogonal basis in $L^{2}(\Omega)$; see [8, Theorem VI.1.4] or [31]. In particular,

$$
E^{-}=\operatorname{span}\left\{e_{1}, \ldots, e_{n^{-}}\right\}, \quad E^{0}=\operatorname{span}\left\{e_{n^{-}+1}, \ldots, e_{\bar{n}}\right\}, \quad E^{+}=\overline{\operatorname{span}\left\{e_{\bar{n}+1}, \ldots\right\}},
$$

where

$$
n^{-}:=\sharp\left\{i: \lambda_{i}<0\right\}, \quad n^{0}:=\sharp\left\{i: \lambda_{i}=0\right\}, \quad \bar{n}:=n^{-}+n^{0} .
$$

Moreover, similar to [31, Lemmas 2.1, 2.3], we have the following lemmas.

Lemma 2.2 Under assumption $V \in L^{N / 2}(\Omega)$ with $N \geq 3$, the norm $\|\cdot\|$ in $E=H_{0}^{1}(\Omega)$ is equivalent to the usual Sobolev norm $\|\cdot\|_{1,2}$ in $H_{0}^{1}(\Omega)$, and $E$ is compactly embedded in $L^{s}(\Omega)$ for $1 \leq s<2 *$. 
Lemma 2.3 Suppose that $V \in L^{N / 2}(\Omega)$ or $(\mathrm{V})$ holds. Then $E$ is compactly embedded in $L^{s}(\Omega)$ for $1 \leq s<2^{*}$, and there exists $\tau_{s}>0$ such that

$$
\|u\|_{s} \leq \tau_{s}\|u\|, \quad \forall u \in E
$$

By (S1) we have

$$
|F(x, u)| \leq c\left(|u|+|u|^{p}\right), \quad \forall(x, u) \in \Omega \times \mathbb{R}
$$

Under (S1) and assumptions of Lemma 2.3, the functional $\Phi$ defined by (1.2) is of class $C^{1}(E, \mathbb{R})$. Moreover, by virtue of (2.3) and (2.6), one has

$$
\Phi(u)=\frac{1}{2}\left(\left\|u^{+}\right\|^{2}-\left\|u^{-}\right\|^{2}\right)-\int_{\Omega} F(x, u) \mathrm{d} x, \quad \forall u \in E,
$$

and

$$
\left\langle\Phi^{\prime}(u), v\right\rangle=\left(u^{+}, v^{+}\right)-\left(u^{-}, v^{-}\right)-\int_{\Omega} f(x, u) v \mathrm{~d} x, \quad \forall u, v \in E .
$$

Before presenting the critical point theorem used in this paper, we give some notions.

Let $X$ be a Banach space and $I \in C^{1}(X, \mathbb{R})$ a functional. A sequence $\left\{u_{n}\right\} \subset X$ is called a (PS) sequence (or $(\mathrm{PS})_{c}$ sequence) if

$$
\left\{I\left(u_{n}\right)\right\} \text { is bounded } \quad\left(\text { or } I\left(u_{n}\right) \rightarrow c\right), \quad I^{\prime}\left(u_{n}\right) \rightarrow 0 .
$$

The functional $I$ is said to satisfy (PS) condition (or (PS) ${ }_{c}$ condition) if each (PS) sequence (or $(\mathrm{PS})_{c}$ sequence) has a convergent subsequence.

A subset $A \subset X$ is said to be symmetric if $u \in A$ implies that $-u \in A$. For a closed symmetric set $A$ which does not contain the origin, we define a genus $\gamma(A)$ of $A$ by the smallest integer $k$ such that there exists an odd continuous mapping from $A$ to $\mathbb{R}^{k} \backslash\{0\}$. If there does not exist such a $k$, we define $\gamma(A)=\infty$. Moreover, we set $\gamma(\emptyset)=0$. Let $\Gamma_{k}$ denote the family of closed symmetric subset $A$ of $X$ such that $0 \notin A$ and $\gamma(A) \geq k$.

Theorem 2.4 ([12, Theorem 1], [16, Theorem 1.1]) Let $X$ be an infinite dimensional Banach space, and $I \in C^{1}(X, \mathbb{R})$ satisfies $(\mathrm{H} 1)$ and $(\mathrm{H} 2)$ below.

(H1) $I$ is even, bounded from below, $I(0)=0$ and I satisfies the (PS) condition;

(H2) for each $k \in \mathbb{N}$, there exists an $A_{k} \in \Gamma_{k}$ such that $\sup _{u \in A_{k}} I(u)<0$.

Then there exists a sequence of critical points $\left\{u_{k}\right\}$ such that $I\left(u_{k}\right) \leq 0, u_{k} \neq 0$ and $\lim _{k \rightarrow \infty} u_{k}=0$.

Remark 2.5 As we see from the proof of [16, Theorem 1.1], the above theorem holds also if we use the (PS) $c$ condition with $c \leq 0$ instead of the (PS) condition in (H1).

Under $V \in L^{N / 2}(\Omega)$ or $(\mathrm{V})$, it follows from (2.5) or (2.7) that $\operatorname{dim}\left(E^{-} \oplus E^{0}\right)<\infty$. We choose an orthonormal basis $\left\{\xi_{j}\right\}_{j=1}^{k_{0}}$ for $E^{-}$, an orthonormal basis $\left\{\xi_{j}\right\}_{j=k_{0}+1}^{l_{0}}$ for $E^{0}$ and an 
orthonormal basis $\left\{\xi_{j}\right\}_{j=l_{0}+1}^{\infty}$ for $E^{+}$, where $k_{0}, l_{0} \in \mathbb{N}$ and $1 \leq k_{0}<l_{0}<\infty$. Then $\left\{\xi_{j}\right\}_{j=1}^{\infty}$ is an orthonormal basis of $E$. Define

$$
X_{j}:=\mathbb{R} \xi_{j}, \quad Y_{k}:=\bigoplus_{j=1}^{k} X_{j}, \quad Z_{k}:=\bigoplus_{j=k+1}^{\infty} X_{j}, \quad k \in \mathbb{Z} .
$$

Proof of Theorem 1.2 Consider the truncated functional

$$
I(u)=\frac{1}{2}\|u\|^{2}-\frac{1}{2}\left(\left\|u^{-}\right\|^{2}+\left\|u^{0}\right\|_{2}^{2}+2 \int_{\Omega} F(x, u) \mathrm{d} x\right) \phi\left(\|u\|^{2}\right), \quad u \in E,
$$

where $\phi: \mathbb{R}^{+} \rightarrow[0,1]$ is a smooth functional such that $\phi(t)=1$ for $t \in[0,1], \phi(t)=0$ for $t \geq 2, \phi^{\prime}(t) \leq 0$ and $\left|\phi^{\prime}(t)\right| \leq 2, \forall t \geq 0$. Obviously, $I \in C^{1}(E, \mathbb{R})$ and $I(0)=0$ by (S2). If we can prove that $I$ satisfies (H1) and (H2), using Theorem 2.4, $I$ admits a sequence of critical points $\left\{u_{k}\right\}$ such that $I\left(u_{k}\right) \leq 0, u_{k} \neq 0$ and $u_{k} \rightarrow 0$ as $k \rightarrow \infty$. So does $\Phi$ by the fact $\Phi(u)=I(u)$ for $\|u\| \leq 1$. Obviously, $I$ is even by (S3)

Note that $I(u)=\frac{1}{2}\|u\|^{2}$ if $\|u\| \geq \sqrt{2}$. Then the functional $I$ is coercive, i.e.

$$
I(u) \rightarrow+\infty, \quad \text { as }\|u\| \rightarrow \infty,
$$

which implies that $I$ is bounded from below and satisfies (PS) $)_{c}$ condition with $c \leq 0$. Indeed, any sequence $\left\{u_{n}\right\} \subset E$ satisfying (2.13) is bounded by (2.16). Passing to a subsequence, we may assume that $u_{n} \rightarrow u$ in $E$. By Lemma 2.3, $u_{n} \rightarrow u$ in $L^{s}(\Omega)$ for $s \in\left[1,2^{*}\right)$, and $u_{n}^{-} \rightarrow u^{-}, u_{n}^{0} \rightarrow u^{0}$ in $E$ since $\operatorname{dim}\left(E^{-} \oplus E^{0}\right)<\infty$. It follows from (S1) and (2.9) that

$$
\begin{aligned}
& \int_{\Omega}\left(\left|f\left(x, u_{n}\right)\right|+|f(x, u)|\right)\left|u_{n}-u\right| \mathrm{d} x \\
& \quad \leq c \int_{\mathbb{R}^{N}}\left(\left|u_{n}\right|+|u|+\left|u_{n}\right|^{p-1}+|u|^{p-1}\right)\left|u_{n}-u\right| \mathrm{d} x \\
& \quad \leq c\left(\left\|u_{n}\right\|_{2}+\|u\|_{2}\right)\left\|u_{n}-u\right\|_{2}+c\left(\left\|u_{n}\right\|_{p}^{p-1}+\|u\|_{p}^{p-1}\right)\left\|u_{n}-u\right\|_{p} \\
& \quad \leq c \tau_{2}^{2}\left(\left\|u_{n}\right\|+\|u\|\right)\left\|u_{n}-u\right\|_{2}+c \tau_{p}^{p-1}\left(\left\|u_{n}\right\|^{p-1}+\|u\|^{p-1}\right)\left\|u_{n}-u\right\|_{p} \\
& \quad=o(1) .
\end{aligned}
$$

By (2.15), direct computation shows that

$$
\begin{aligned}
\left\langle I^{\prime}(u), v\right\rangle= & {\left[1-\left(\left\|u^{-}\right\|^{2}+\left\|u^{0}\right\|_{2}^{2}+2 \int_{\Omega} F(x, u) \mathrm{d} x\right) \phi^{\prime}\left(\|u\|^{2}\right)\right](u, v) } \\
& -\left[\left(u^{-}, v^{-}\right)+\left(u^{0}, v\right)_{L^{2}}+\int_{\Omega} f(x, u) v \mathrm{~d} x\right] \phi\left(\|u\|^{2}\right), \quad u, v \in E .
\end{aligned}
$$

It follows from (2.13) and (2.15) that

$$
-\left(\left\|u_{n}^{-}\right\|^{2}+\left\|u_{n}^{0}\right\|_{2}^{2}+2 \int_{\Omega} F\left(x, u_{n}\right) \mathrm{d} x\right) \phi\left(\left\|u_{n}\right\|^{2}\right)=2 I\left(u_{n}\right)-\left\|u_{n}\right\|^{2} \leq 0,
$$

which implies that

$$
\left\|u_{n}^{-}\right\|^{2}+\left\|u_{n}^{0}\right\|_{2}^{2}+2 \int_{\Omega} F\left(x, u_{n}\right) \mathrm{d} x \geq 0
$$


Note that $\phi^{\prime}(t) \leq 0$ and $\left|\phi^{\prime}(t)\right| / 2, \phi(t) \leq 1$ for any $t \geq 0$, then, by (2.9), (2.17), (2.18) and (2.19), one has

$$
\begin{aligned}
\| u_{n}- & u \|^{2} \\
= & \left\langle I^{\prime}\left(u_{n}\right)-I^{\prime}(u), u_{n}-u\right\rangle+\left(\left\|u_{n}^{-}\right\|^{2}+\left\|u_{n}^{0}\right\|_{2}^{2}+2 \int_{\Omega} F\left(x, u_{n}\right) \mathrm{d} x\right) \\
& \quad \times \phi^{\prime}\left(\left\|u_{n}\right\|^{2}\right)\left(u_{n}, u_{n}-u\right)-\left(\left\|u^{-}\right\|^{2}+\left\|u^{0}\right\|_{2}^{2}+2 \int_{\Omega} F(x, u) \mathrm{d} x\right) \phi^{\prime}\left(\|u\|^{2}\right)\left(u, u_{n}-u\right) \\
& +\phi\left(\left\|u_{n}\right\|\right)\left[\left\|u_{n}^{-}-u^{-}\right\|^{2}+\left\|u_{n}^{0}-u^{0}\right\|_{L^{2}}^{2}+\int_{\Omega}\left(f\left(x, u_{n}\right)-f(x, u)\right)\left(u_{n}-u\right) \mathrm{d} x\right] \\
& +\left[\phi\left(\left\|u_{n}\right\|\right)-\phi(\|u\|)\right]\left[\left(u^{-}, u_{n}^{-}-u^{-}\right)+\left(u^{0}, u_{n}^{0}-u^{0}\right)_{L^{2}}+\int_{\Omega} f(x, u)\left(u_{n}-u\right) \mathrm{d} x\right] \\
\leq & o(1)+\left(\left\|u_{n}^{-}\right\|^{2}+\left\|u_{n}^{0}\right\|_{2}^{2}+2 \int_{\Omega} F\left(x, u_{n}\right) \mathrm{d} x\right) \phi^{\prime}\left(\left\|u_{n}\right\|^{2}\right)\left(u_{n}, u_{n}-u\right) \\
& +\int_{\Omega}\left(\left|f\left(x, u_{n}\right)\right|+|f(x, u)|\right)\left|u_{n}-u\right| \mathrm{d} x+2 \int_{\Omega}|f(x, u)|\left|u_{n}-u\right| \mathrm{d} x \\
= & o(1)+\left(\left\|u_{n}^{-}\right\|^{2}+\left\|u_{n}^{0}\right\|_{2}^{2}+2 \int_{\Omega} F\left(x, u_{n}\right) \mathrm{d} x\right) \phi^{\prime}\left(\left\|u_{n}\right\|^{2}\right)\left\|u_{n}-u\right\|^{2} \\
& +\left(\left\|u_{n}^{-}\right\|^{2}+\left\|u_{n}^{0}\right\|_{2}^{2}+2 \int_{\Omega} F\left(x, u_{n}\right) \mathrm{d} x\right) \phi^{\prime}\left(\left\|u_{n}\right\|^{2}\right)\left(u, u_{n}-u\right) \\
= & o(1)+\left(\left\|u_{n}^{-}\right\|^{2}+\left\|u_{n}^{0}\right\|_{2}^{2}+2 \int_{\Omega} F\left(x, u_{n}\right) \mathrm{d} x\right) \phi^{\prime}\left(\left\|u_{n}\right\|^{2}\right)\left\|u_{n}-u\right\|^{2} \\
\leq & o(1) .
\end{aligned}
$$

Thus $I$ satisfies the $(\mathrm{PS})_{c}$ condition with $c \leq 0$.

For any fixed $l_{0}+1 \leq k \in \mathbb{N}$, where $l_{0}=\operatorname{dim}\left(E^{-} \oplus E^{0}\right)<\infty$. By (2.14) and the equivalence of the norms on finite dimensional spaces, there exist constants $c_{k}, d_{k}>0$ such that

$$
\|u\|_{2} \geq c_{k}\|u\|, \quad \operatorname{ess} \sup _{x \in \Omega}|u(x)|:=\|u\|_{\infty} \leq d_{k}\|u\|, \quad \forall u \in Y_{k}
$$

(S2) implies the existence of a constant $r \in(0,1)$ such that $F(x, u) \geq c_{k}^{-2}|u|^{2}$ for all $|u| \leq r$ and a.e. $x \in \Omega$. Then, for any $u \in Y_{k}$ with $\|u\|=l_{k}:=2^{-1} \min \left\{1, r d_{k}^{-1}\right\}$, one has

$$
\begin{aligned}
I(u) & =\frac{1}{2}\left\|u^{+}\right\|^{2}-\frac{1}{2}\left\|u^{-}\right\|^{2}-\int_{\Omega} F(x, u) \mathrm{d} x \\
& \leq \frac{1}{2}\left\|u^{+}\right\|^{2}-\int_{\Omega} F(x, u) \mathrm{d} x \leq \frac{1}{2}\|u\|^{2}-\frac{1}{c_{k}^{2}}\|u\|_{2}^{2} \\
& \leq \frac{1}{2}\|u\|^{2}-\frac{c_{k}^{2}}{c_{k}^{2}}\|u\|^{2}=-\frac{1}{2}\|u\|^{2} \\
& =-\frac{1}{2} l_{k}^{2},
\end{aligned}
$$

which implies that

$$
\left\{u \in Y_{k}:\|u\|=l_{k}\right\} \subset\left\{u \in E: I(u) \leq-\frac{1}{2} l_{k}^{2}\right\} .
$$


Let $A_{k}:=\left\{u \in E: I(u) \leq-2^{-1} l_{k}^{2}\right\}$. Then

$$
\gamma\left(A_{k}\right) \geq \gamma\left(\left\{u \in Y_{k}:\|u\|=l_{k}\right\}\right) \geq k .
$$

Clearly $A_{k} \in \Gamma_{k}$ and $\sup _{u \in A_{k}} I(u) \leq-2^{-1} l_{k}^{2}<0$. Then we get the result from Theorem 2.4.

Funding

This work is partially supported by the NSFC (Nos: 11971485, 11801574) of China, Natural Science Foundation of Hunan Province (No. 2019JJ50788) and Central South University Innovation-Driven Project for Young Scholars (No. 2019CX022).

\section{Availability of data and materials}

This paper focuses on theoretical analysis, not involving experiments and data.

\section{Competing interests}

The authors declare that they have no competing interests.

\section{Authors' contributions}

The authors have equal contributions to each part of this paper. All authors read and approved the final manuscript.

\section{Author details}

${ }^{1}$ School of Mathematics and Statistics, Central South University, Changsha, 410083 Hunan, P.R. China. ${ }^{2}$ School of Traffic and Transportation Engineering, Central South University, Changsha, 410075 Hunan, P.R. China.

\section{Publisher's Note}

Springer Nature remains neutral with regard to jurisdictional claims in published maps and institutional affiliations.

Received: 13 August 2020 Accepted: 30 September 2020 Published online: 07 October 2020

\section{References}

1. Ambrosetti, A., Brezis, H., Cerami, G.: Combined effects of concave and convex nonlinearities in some elliptic problems. J. Funct. Anal. 122, 519-543 (1994)

2. Ambrosetti, A., Rabinowitz, P.H.: Dual variational methods in critical point theory and applications. J. Funct. Anal. 14 349-381 (1973)

3. Amster, P.: Multiple solutions for an elliptic system with indefinite Robin boundary conditions. Adv. Nonlinear Anal. 8, 603-614 (2019)

4. Chen, J., Tang, X.H.: Infinitely many solutions for a class of sublinear Schrödinger equations. Taiwan. J. Math. 19, 381-396 (2015)

5. Costa, D.G.: Variational problems which are nonquadratic at infinity. In: Brezis, H. (ed.) Morse Theorem, Minimax Theory and Applications to Nonlinear Differential Equations. International Press, Somerville (2003)

6. Costa, D.G., Magalhães, C.A.: Variational elliptic problems which are nonquadratic at infinity. Nonlinear Anal. 23 1401-1412 (1994)

7. Ding, Y.H.: Infinitely many entire solutions of an elliptic system with symmetry. Topol. Methods Nonlinear Anal. 9, 313-323 (1997)

8. Edmunds, D., Evans, W.: Spectral Theorem and Differential Operators. Clarendon, Oxford (1987)

9. He, W., Qin, D.D., Wu, Q.F.: Existence, multiplicity and nonexistence results for Kirchhoff type equations. Adv. Nonlinear Anal. 10 (2021). https://doi.org/10.1515/anona-2020-0154

10. He, X., Zou, W.: Multiplicity of solutions for a class of elliptic boundary value problems. Nonlinear Anal. 71, 2606-2613 (2009)

11. Jiang, Q., Tang, C.: Existence of a nontrivial solution for a class of superquadratic elliptic problems. Nonlinear Anal. 69 , 523-529 (2008)

12. Kajikiya, R.: A critical point theorem related to the symmetric mountain pass lemma and its applications to elliptic equations. J. Funct. Anal. 225, 352-370 (2005)

13. Ke, X.-F., Tang, C.-L.: Existence and multiplicity of solutions to semilinear elliptic equation with nonlinear term of superlinear and subcritical growth. Electron. J. Differ. Equ. 2018, 88 (2018)

14. Li, L., Tang, C.-L.: Infinitely many solutions for resonance elliptic systems. C. R. Math. Acad. Sci. Paris 353, 35-40 (2015)

15. Li, S., Willem, M.: Applications of local linking to critical theory. J. Math. Anal. Appl. 189, 6-32 (1995)

16. Liu, Z.L., Wang, Z.-Q.: On Clark's theorem and its applications to partially sublinear problems. Ann. Inst. Henri Poincaré, Anal. Non Linéaire 32, 1015-1037 (2015)

17. Ouyang, T.-C., Shi, J.-P.: Exact multiplicity of positive solutions for a class of semilinear problem. J. Differ. Equ. 146 121-156 (1998)

18. Pan, H.-L., Tang, C.-L.: Existence of infinitely many solutions for semilinear elliptic equations. Electron. J. Differ. Equ. 2016, 167 (2016)

19. Papageorgiou, N.S., Rădulescu, V.D., Repovš, D.D.: Double-phase problems with reaction of arbitrary growth Z. Angew. Math. Phys. 69(4), Paper No. 108 (2018)

20. Papageorgiou, N.S., Rădulescu, V.D., Repovš, D.D.: Nonlinear Analysis-Theory and Methods. Springer Monographs in Mathematics. Springer, Cham (2019) 
21. Papageorgiou, N.S., Rădulescu, V.D., Repovš, D.D.: Nonlinear nonhomogeneous singular problems. Calc. Var. Partial Differ. Equ. 59(1), Paper No. 9 (2020)

22. Qin, D.D., Chen, J., Tang, X.H.: Existence and non-existence of nontrivial solutions for Schrödinger systems via Nehari-Pohozaev manifold. Comput. Math. Appl. 74(12), 3141-3160 (2017)

23. Qin, D.D., Tang, X.H., Wu, Q.F.: Ground states of nonlinear Schrödinger systems with periodic or non-periodic potentials. Commun. Pure Appl. Anal. 18(3), 1261-1280 (2019)

24. Qin, D.D., Tang, X.H., Wu, Q.F.: Existence and concentration properties of ground state solutions for elliptic systems. Complex Var. Elliptic Equ. 65(8), 1257-1286 (2020)

25. Qin, D.D., Tang, X.H., Zhang, J.: Multiple solutions for semilinear elliptic equations with sign-changing potential and nonlinearity. Electron. J. Differ. Equ. 2013, 207 (2013)

26. Rabinowitz, P.H.: Minimax Methods in Critical Point Theory with Applications to Differential Equations. CBMS Reg. Conf. Ser. in Math., vol. 65. Am. Math. Soc., Providence (1986)

27. Rolando, S.: Multiple nonradial solutions for a nonlinear elliptic problem with singular and decaying radial potential. Adv. Nonlinear Anal. 8, 885-901 (2019)

28. Szulkin, A., Weth, T.: The method of Nehari manifold. In: Gao, D.Y., Motreanu, D. (eds.) Handbook of Nonconvex Analysis and Applications, pp. 597-632. International Press, Boston (2010)

29. Tang, X.H.: Non-Nehari manifold method for superlinear Schrödinger equation. Taiwan. J. Math. 18, 1957-1979 (2014)

30. Willem, M.: Minimax Theorems. Birkhäuser, Boston (1996)

31. Zhang, Q.Y., Liu, C.G.: Multiple solutions for a class of semilinear elliptic equations with general potentials. Nonlinear Anal. 75, 5473-5481 (2012)

32. Zhang, W., Tang, X.H., Zhang, J.: Infinitely many solutions for elliptic boundary value problems with sing-changing potential. Electron. J. Differ. Equ. 2014, 53 (2014)

33. Zou, W.: Variant fountain theorems and their applications. Manuscr. Math. 104, 343-358 (2001)

\section{Submit your manuscript to a SpringerOpen ${ }^{\circ}$ journal and benefit from:}

- Convenient online submission

- Rigorous peer review

- Open access: articles freely available online

- High visibility within the field

- Retaining the copyright to your article

Submit your next manuscript at $\boldsymbol{\nabla}$ springeropen.com 